\title{
Universal and Environmental Influences on Star Clusters
}

\author{
Bradley C. Whitmore ${ }^{1}$ \\ ${ }^{1}$ Space Telescope Science Institute, 3700 San Martin Drive, Baltimore, MD 21218, USA \\ email: whitmore@stsci.edu
}

\begin{abstract}
There is now evidence that some aspects of compact star cluster formation and destruction are quasi-universal in nature, and some aspects depend on environment. But what do we mean by these terms, environmental and universal? Is one the dominant influence? How can things be both universal and environmentally dependent? In this contribution we first provide a brief historical overview, then examine evidence for both universality and environmental dependences, and finish by examining a new approach that both demonstrates the degree to which cluster mass functions are universal (i.e., to a level of roughly 0.2 in the Log over three orders of magnitude when normalizing by the star formation rate), and enables a method for quantifying 2nd-order environmental effects.
\end{abstract}

\section{Introduction - A Brief History and the Size-of-Sample Effect}

One of the first major results from the Hubble Space Telescope, even while in its aberrated state, was the discovery of Super Star Clusters (SSCs; fainter clusters are often called Young Massive Clusters, YMCs) in merging galaxies (e.g., NGC 1275 - Holtzman et al. (1992), NGC 7252 - Whitmore et al. (1993), the Antennae - Whitmore et al. (1995)). The implication that we could study the formation of young globular clusters in the local universe rather than trying to ascertain how they formed 13 billion years ago caught the interest of a generation of astronomers. An obvious inference was that there was something special about the environment of interacting galaxies that allowed SSCs to form in these systems and no others. This turned out not to be the case.

YMCs were also found in a variety of other types of galaxies, ranging from starburst (NGC 1569 and NGC 1705 -O'Connell et al. (1994)), to barred galaxies (NGC 1097 \& NGC 6951 -Barth et al. (1995)). The finding by Larsen \& Richtler (1999) that YMCs could also be found in spiral galaxies, albeit generally with fainter luminosities and lower masses, was another important early discovery. These results suggested that the formation of young massive clusters was more universal than first thought.

The first compilation of cluster luminosity functions for merging galaxies was made by Whitmore (2003). Because the sample was small, a simpler statistic, the luminosity of the brightest cluster versus the Log of the number of clusters in the galaxy brighter than $\mathrm{Mv}=-9$ (i.e., Log N) was also plotted. The expectation was that the distribution would be bi-modal, with the spirals and dwarf galaxies having fainter characteristic luminosities and the mergers having brighter characteristic magnitudes. The surprising result was the finding that a well defined, continuous linear correlation was present. It soon became evident that this relationship could be explained if all the galaxies had the same luminosity function with $d N / d L \propto L^{-2}$ (see Figure 1). It appears that the brightest clusters in merging galaxies are brighter because they come from a larger sample, not because there is necessarily any special physics involved. The larger sample of clusters in these galaxies fills out the distribution more completely, leading to a population of very 


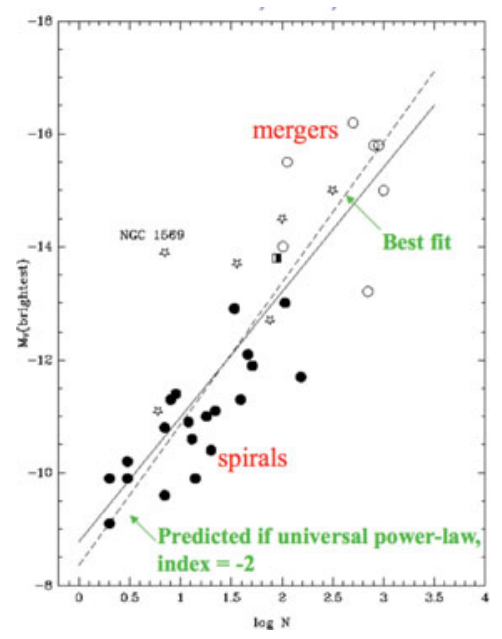

Figure 1. $M_{V \text { (brightest) }}$ vs. Log $\mathrm{N}$ from Whitmore (2003).

bright, massive clusters, along with very large numbers of fainter, less massive clusters. This is generally called the "size-of-sample" effect (De Vaucouleurs (1970)).

A number of other studies found similar results, including Larsen et al. (2002), Bastian (2008), Whitmore et al. (2014), and Adamo et al. (2015). The relationship has now been extended over six orders of magnitudes, from dwarf galaxies (Bastian (2008)) to ULIRGS (Vavilkin (2011)). The use of Log SFR (Star Formation Rate) on the X axis stretches out the diagram by allowing the inclusions of very faint dwarf galaxies, which generally do not have clusters brighter than $\mathrm{Mv}=-9$. The similarity between the versions of the diagram using Log $\mathrm{N}$ and using Log SFR, and the tight correlation between Log $\mathrm{N}$ and Log SFR (e.g., RMS scatter $=0.11$ in Figure 7 in Whitmore et al. (2014)) shows that Log SFR is a good stand-in for the sample size, a point that will be important in $\S 5$.

Hence, it appears that there is a roughly universal luminosity function for young clusters (see Whitmore et al. (2014) for additional discussion). The larger sample (higher SFR) in mergers may simply be due to the fact that conditions for making clusters are globally present in mergers, presumably due to the higher pressure of the ISM due to the collision, but only locally present in the arms of spiral galaxies.

\section{What Does Universality Mean and Why Do We Care?}

What do we mean by Universality? In the context used in this paper, it means the fundamental relationships, e.g., the luminosity function, the mass function, and the age distribution, are the same for all star-forming galaxies in all environments, once the size-of-sample effects are taken into account.

Taken to extremes, it is impossible for anything to be perfectly universal, since this represents a limiting case that can never be reached. As soon as there is a deviation, at the $10 \%$, or $1 \%$, or $0.01 \%$ level, perfect universality breaks down. What we are really interested in is what are the dominant influences that determine the star cluster demographics. For this reason we use the term "quasi-universal", rather than using the extreme definition.

Why do we care? If the dominant influences are quasi-universal (e.g., deviations are a few tenths over several orders of magnitude), this implies that the primary physical influences are internal to the cluster (e.g., gas loss, stellar evolution, stellar 

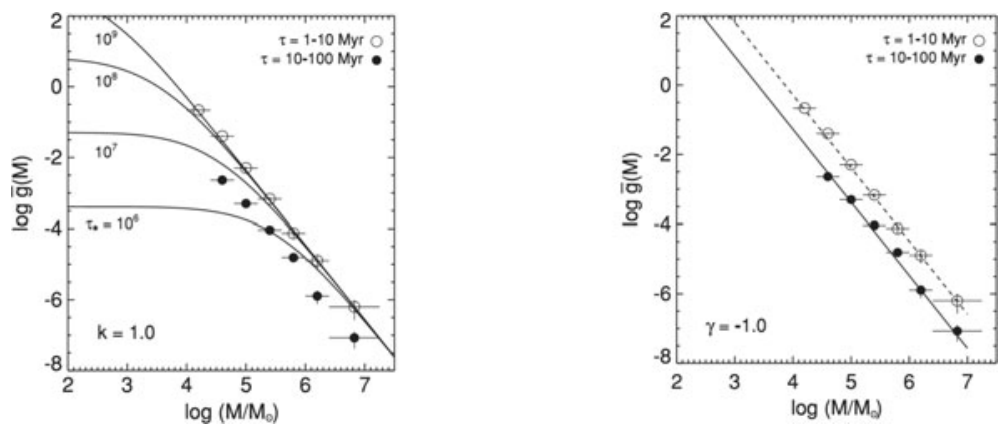

Figure 2. Predicted mass functions from environmental models (left) and the quasi-universal model (right) overlaid on data from the Antennae galaxies. From Fall et al. (2009).

ejection, ...). If the dominant influences are environmental, this implies that the primary physical influences are external to the clusters; for example the position in the galaxy (and hence the interstellar pressure, the density of GMCs, the tidal shear), or the morphology of the galaxy itself (e.g., merger, spiral, dwarf).

\section{Straight or Curved Lines ?}

Papers that have discussed the universal model include Whitmore et al. (2004), Whitmore et al. (2007), Lamers (2009), Fall et al. (2009), and Fall \& Chandar (2012).

A very brief, overly simplified, phenomenological description of the difference between the quasi-universal model and the environmentally dependent model is that the former predicts straight lines in the mass function and age distribution diagrams, while the later predicts curved lines.

Figure 2 shows an example using data from the Antennae galaxies in Fall et al. (2009). While the data appears to produce nearly linear mass functions, as predicted by the quasi-universal model as shown in the right panel, various environmental models predict curved lines, as shown in the left diagram. If the dissipation time is very long (e.g., $10^{9}$ yrs), the predicted line is nearly linear, but the offset between the two age ranges is still not predicted. Similar results for the age distributions can be found in Figure 11 from Fall et al. (2009).

Observational incompleteness also produces curvature, and can mimic environmental effects. A linear relationship, as found in the data, represents a limiting case and is not likely to be the result of some conspiracy between completeness issue, selection effects, or other observational difficulties, all of which typically result in non-linear relations.

Cumulative fits can be useful for showing deviations from linearity. However, they also amplify noise from small number statistics. Several studies by proponents of environmental models employ cumulative fits (e.g., Figs. 9 and 15 from Bastian et al. (2012)).

One approach is to show results using both normal binning and cumulative fits, to allow readers to develop a better feel for the degree and reliability of possible deviations. An example where both techniques are shown is Whitmore et al. (2014) (e.g., Figures 5 and 9 ). Note the $1 \%$ arrows in Figure 9 that reminds the reader that these deviations from linearity in a cumulative fit often reflect a very small number of clusters.

\section{Recent Evidence of Environmental Effects}

Papers that have explored environmental models include Boutloukos, \& Lamers (2003), Lamers (2009), Bastian et al. (2012), Kruijssen et al. (2012), and Adamo et al. (2015). 

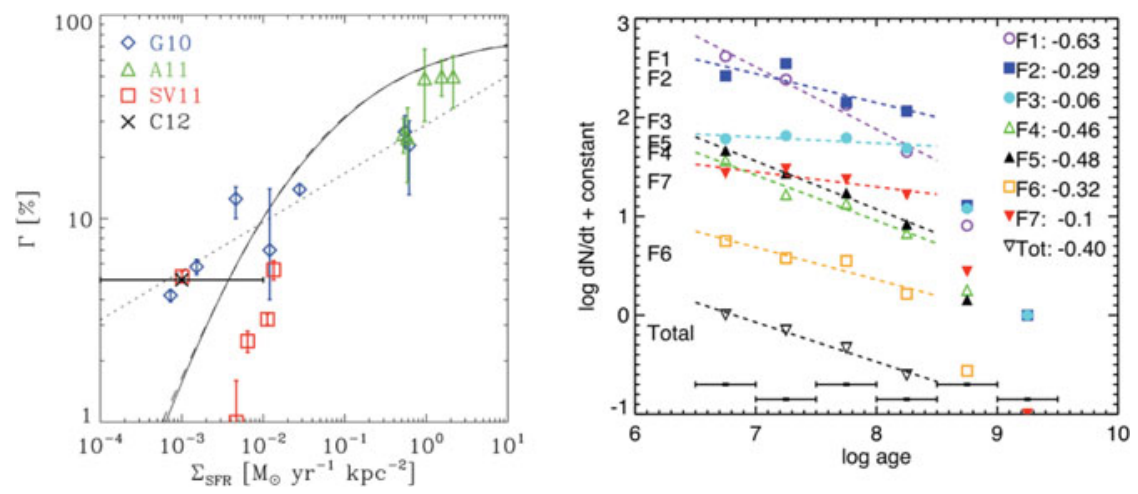

Figure 3. Examples of environmental dependencies. Left - Relationship between the fraction of mass in bound clusters, $\Gamma$, vs Specific Star Formation Rate (from Kruijssen et al. (2012)). Right - Cluster age distributions for seven fields in M83 (from Silva-Villa et al. (2014)).

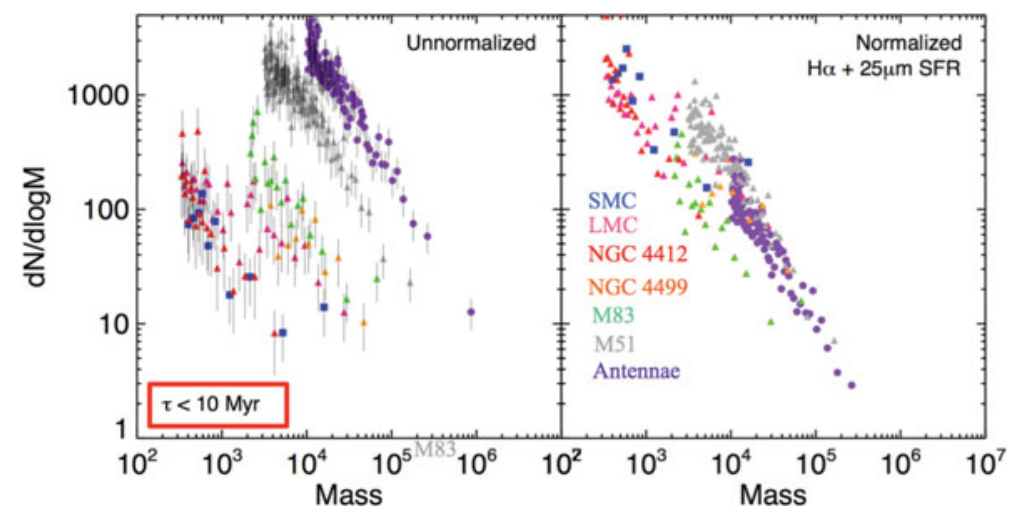

Figure 4. Original (left) and normalized (right) Cluster Mass Functions for clusters with ages less than 10 Myr (from Chandar et al. (2015)).

Figure 3 shows two of the most convincing recent examples of environmental dependencies. In the left panel we find both theoretical (dashed lines - Kruijssen et al. (2012)) and observational (dotted line and data points from Goddard et al. (2010)) indications that the fraction of mass in bound clusters, $\Gamma$, depends on the specific Star Formation Rate. While the determination of $\Gamma$ is rather elaborate, involving a number of measurements (e.g., SFR, field of view used for SFR), assumptions (slope of mass function = -2.0 , cluster age range of $7 \mathrm{Myr}$ ) and extrapolations (to lower mass clusters in order to determine the total population of clusters), the effect does appear to be real.

In the right panel we find evidence that the slope of the age distribution in the spiral galaxy M83 flattens in the outer parts of the galaxy (Silva-Villa et al. (2014)). While there appears to be a relatively clear trend with radius (see paper for details), we note that the mean slope is -0.4 . This represents a $60 \%$ disruption rate per decade. We also note that the relationships are all relatively straight in the region of completeness (to $10^{8.5} \mathrm{yrs}$ ), with downturns due to incompleteness beyond this range.

\section{A New Result - CMF/SFR for Seven Galaxies}

An underlying implication of the quasi-universal model is that the Star Formation Rate (SFR) and cluster formation rate are directly proportional. If so, it should be possible 


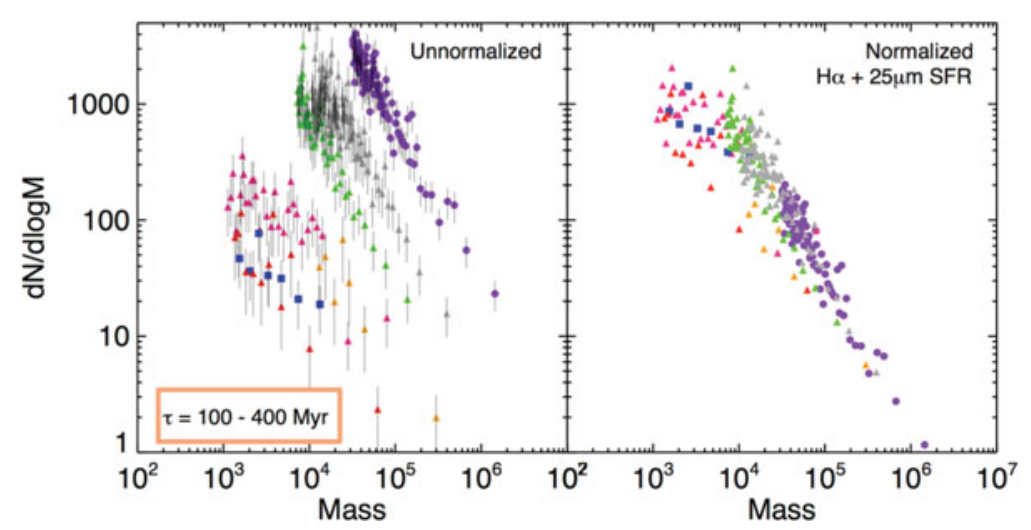

Figure 5. Same as Figure 4 for an older sample of clusters.
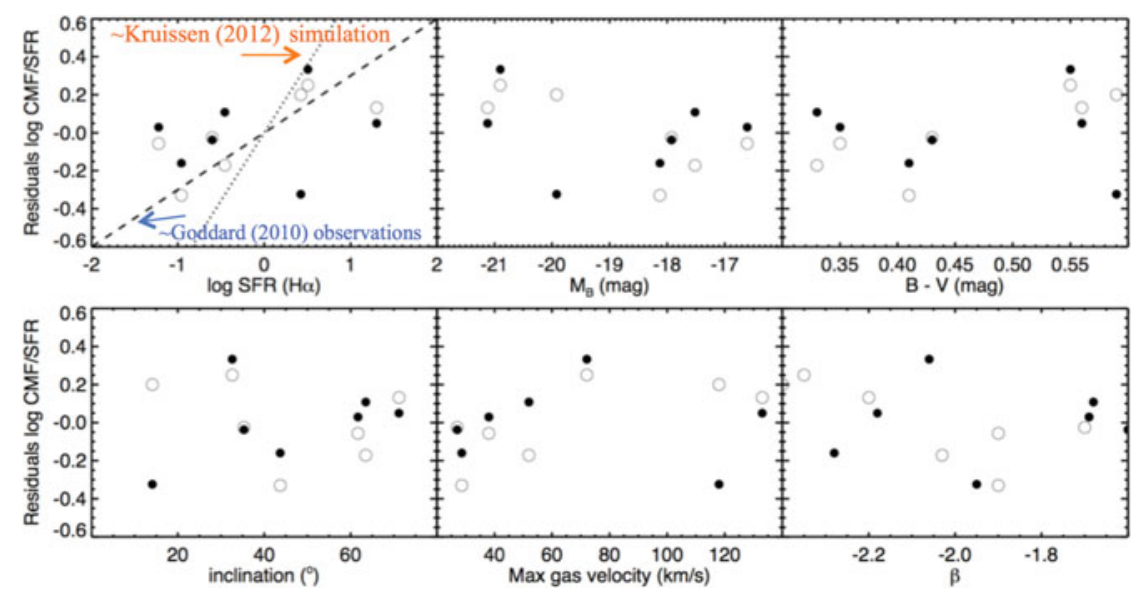

Figure 6. From Chandar et al. (2015). See text for discussion.

to use the SFR (e.g., based on extinction corrected Halpha) to scale the Cluster Mass Function (CMF) of all galaxies to fit on the same universal relation. Figure 4 (from Chandar et al. (2015) - hereafter CFW) shows that normalizing by SFR collapses the spread from three orders of magnitude to a RMS scatter of a few tenths in the Log.

Somewhat surprisingly, Figure 5 shows that this works just as well for an older population of clusters, where we can be certain that the clusters are 'bound', an issue of some debate when working with clusters less than 10 Myr old.

The scatter in both diagrams is $\approx 0.2$ in the Log, roughly the same as expected from the error budget. This suggests that the SFR has been relatively constant over a long period of time, and the disruption rate must also be relatively universal.

Are there any clear 2nd-order correlations? It appears that the dwarf galaxies (e.g., NGC 4412, SMC, LMC) remain slightly low in the two diagrams after normalization, perhaps reflecting a smaller bound fraction of clusters. Figure 6 shows the residuals from the normalized diagrams for a variety of parameters. Focusing on the relationship for Log SFR in the upper left panel, we find that the relationship for the 10 Myr sample (solid points) is not statistically significant. For the older populations the relationship is only significant at the $93 \%$ level (i.e., about a $2 \sigma$ result). However, we note that if there is a trend, it is in the same sense as the relationship with $\Gamma$ from Kruijssen et al. (2012), although not as steep, especially with respect to the simulation. 
Linear approximations of the Kruijssen et al. (2012) simulation, and the observational trend from Goddard et al. (2010), are included in Figure 6 (i.e., by assuming the same range in $\Gamma$ from the faintest to brightest galaxies in both samples). Note that the Kruijssen diagram uses the Specific SFR (hereafter SSFR - i.e., divided by the surface area of the galaxy), while the CFW figure uses Log SFR. This reflects our focus on examining the degree of universality, by using a parameter that is closely related to the sample size (i.e., Log SFR - see discussion in §1) to normalize the CMFs. Kruijssen et al. (2012) is primarily interested in the environmental dependence, hence they employ the SSFR rate as the independent variable to remove the size-of-sample (universality) effect.

Both approaches have their merits. As recommended in $\S 3$, it is often advantageous to try more than one approach. In this way we can better ascertain to what degree universality can explain the range in different CMFs, and to what degree environmental dependencies are important.

While the results using the CMF/SFR approach look promising, we should keep in mind that the sample is only seven galaxies. The Large Extragalactic Galaxies UV Survey (LEGUS - Calzetti et al. (2015)) sample will provide a much larger sample.

\section{References}

Adamo, A., Kruijssen, J. M. D., Bastian, N., Silva-Villa, E., \& Ryon, J. 2015, MNRAS, 452, 246

Barth, A. J., Ho, L. C., Filippenko, A. V., \& Sargent, W. L. 1995, AJ, 110, 1009

Bastian, N. 2008, MNRAS, 390, 759

Bastian, N., Adamo, A., Gieles, M., et al. 2012, MNRAS, 419, 2606

Boutloukos, S. G. \& Lamers, H. J. G. L. M. 2003, MNRAS, 338, 717

Calzetti, D., Lee, J. C., Sabbi, E., et al. 2015, AJ, 149, 51

Chandar, R., Fall, S. M., \& Whitmore, B. C. 2015, ApJ, 810, 1

De Vaucouleurs, G. 1970, ApJ, 159, 435

Fall, S. M. \& Chandar, R. 2012, ApJ, 752, 96

Fall, S. M., Chandar, R., \& Whitmore, B. C., 2009, ApJ, 704, 453

Goddard, Q. E., Bastian, N., \& Kennicutt, R. C. 2010, MNRAS, 405, 857

Holtzman, J. A. et al. 1992, AJ, 103, 69

Kruijssen, J. M. D. 2012, MNRAS, 426, 3008

Lamers, H. J. G.. L. M.. 2009, Ap\&SS, 324,183

Larsen, S. S. 2002, AJ, 124, 1393

Larsen, S. S. \& Richtler, T. 1999, A\&A, 345, 59

O'Connell, R. W., Gallagher, J. S., \& Hunter, D. A. 1994, ApJ, 433, 650

Silva-Villa, E., Adamo, A., Bastian, N., Fouesneau, M., \& Zackrisson, E. 2014, MNRAS, 440, 116

Vavilkin, T. 2011, PhD thesis, State University of New York at Stony Brook

Whitmore, B. C. 2003, in A Decade of Hubble Space Telescope Science, ed. Mario Livio, Keith Noll \& Massimo Stiavelli (Cambridge, UK: Cambridge Univ. Press)

Whitmore, B. C. 2004, in ASP Conf. Ser. 322, The Formation and Evolution of Massive Young Star Clusters, ed. H. Lamers, L. J. Smith, \& A. Nota (San Francisco: ASP), 419

Whitmore, B. C., et al. 2014, AJ, 147, 78

Whitmore, B. C., Chandar, R., \& Fall, S. M. 2007, AJ, 133, 1067

Whitmore, B. C. \& Schweizer, F. 1995, AJ, 109, 960

Whitmore, B. C., Schweizer, F., Leitherer, C., Borne, K., \& Robert, C. 1993, AJ, 106, 1354 